\title{
Hospital Information System: Closed-loop Application Based on Radio Frequency Identification
}

\author{
Hong Wang \\ Information Department of 309 Hospital \\ No.17th Heishanhu Road, Haidian District \\ Beijing, China \\ e-mail:wh70358@126.com
}

\author{
Yuanli Wu \\ Information Department of 309 Hospital \\ No.17th Heishanhu Road, Haidian District \\ Beijing, China \\ e-mail: dtnudt@126.com
}

\begin{abstract}
To prevent medical errors or even accidents, our solution is to design the closed-loop application based on Radio Frequency Identification (RFID) technology in the Hospital Information System (HIS). We focus on the information improvement for doctor's order based on RFID. The whole closed-loop process starts from the initial collection of the patient's information, through confirmation of the unique identification of the patient, and to the finishing of the doctor's order at last. The final information is written back into HIS. Medical staff can collect and deal with the patient's information straightly through the system, thereby guarantee maximal medical information correctness. We present the overall system design of the closed-loop process of the doctor's order, which includes workflow realization and the wireless system architecture based on RFID in the ward environment.
\end{abstract}

Keywords-medical error;RFID;HIS;closed-loop;wireless architecture

\section{INTRODUCTION}

Improving patient's safety is an arduous task. So we need to provide the medical application with its ability to help provide key service to reduce medical errors effectively.

It is now an open-loop process from the initial giving of the doctor's order to the final completion of it. The loop is not closed and has many intermediate links in the process. Even though the doctor's order is saved in the Hospital Information System (HIS), sometimes we don't know who actually executed it, and when it was executed [1]. The traditional checking and identify method is not easy to be guaranteed during the process [2]. The more open-loop the medical process is, the more difficult it is to reduce medical errors. Therefore the open-loop system must be changed to a closed one [3].

Our research aims at realizing the closed-loop process of the doctor's order to reduce medical errors by applying Radio Frequency Identification (RFID) technology in HIS. The goal is to enable RFID technology to meet the need of the special hospital environment, include system structure, system compatibility with existing HIS, etc.

The rest of this paper is organized as follows. Section 2 introduces related wireless architecture and RFID technology, describes the composition of wireless network and how RFID works in order to apply them in HIS. Section 3 gives an overview of the system design, and provides a detailed realization on architecture of the wireless system based on
RFID in ward environment. Section 4 presents the realization of wireless nursing system, include the workflow and function module. Finally Section 5 concludes this research with the summary.

\section{BACKGROUD}

\section{A. Wireless Architecture Compared}

Wireless network uses radio wave as the medium to transmit information and constitutes Wireless Local Area Network (WLAN). Wireless network can provide free moving and roaming service for their users within its cover range, thus user will not be tied up to the cable network.

Wireless structured network is composed of Access Point (AP), Station (STA) and Distribute System Set (DSS). The covered area falls into Basic Service Area and Extended Service Area. The wireless AP is also called the wireless Hub, which is used for receiving, caching and transmitting data between wireless STA and the cable network. The wireless AP can cover dozens of or even hundreds of users, and the radius can reach more than one hundred meters.

The wireless AP and wireless STA form the Basic Service Set (BSS). AP and its STA are interrelated at any time. In other words, a cellular area covered by one AP is called Basic Service Area. The wireless AP and wireless STA are interrelated by the Basic Service Identifier of AP.

To meet moving need from patients and medical staff, wireless architecture is selected to the closed-loop HIS based on RFID.

\section{B. RFID Technology}

RFID stands for Radio Frequency Identification. It uses electromagnetic induction or electromagnetic transmission to communicate. Using the sequential data relationships within the communication link, energy and data transmission achieved, and thus fulfill the goal of identification and tracking of non-contact objects.

The basic working method of the RFID system is to install RFID tags on the identified objects (by pasting, plugging up, wearing and implanting etc.). When the object which is identified by the RFID reader entered into the reading range, the wireless communication link between the tag and reader is established, and the tag sends information of itself to the reader, such as tag number and other data stored etc. The reader receives the message and decodes before transmits it to the background computer to process, 
and then complete the whole information processing procedure.

RFID reader which does not need cable is based on wireless network. At the same time RFID tag has another feature that allows information in the tag to be updated through wireless network. RFID reader can scan multiple tags simultaneously, comparing to the bar coder that has to be read by corresponding scanner one by one in sequence. Bar coder of line type can only accommodate 10 to 20 characters of data. In contrast RFID tag can store thousands of character bytes. In addition RFID tag can be cleaned by alcohol. It will not have the situation as the bar code, which cannot be read in the humid environment, or can be contaminated by blood or cannot be used when it is worn and not readable. So RFID is more convenient to be applied in the medical area $[4,5,6]$.

Our research aims at adopting the RFID technology to build the information closed-loop system as follows.

\section{CONSTRUCT WIRELESS ClOSED-LOOP SYSTEM BASED ON RFID}

\section{A. Wireless Network Structure}

First We map out the Hospital Wireless Network. The hospital user needs to access devices or services of cable network. According to this fact, the ESS network structure is selected. By interconnecting with different BSS in ESS sharing by AP, related wireless terminals, such as Personnel Digital Assistance (PDA), Tablet PC and other wireless device, fall in the same segment. In the application layer Dynamic Host Configuration Protocol (DHCP) is applied. Every wireless terminal obtains its own IP automatically. Figure 1 show the hospital wireless network structure.

Related wireless standards are IEEE 802.11b, IEEE 802.11g, IEEE 802.11h and IEEE 802.11n, etc. Difference between them is mainly the transmission speed. Now IEEE 802.11b is most common used. But IEEE 802.11g has more possibility to become the next generation standard. New standard such as IEEE 802.11n is now in rapid development period.

\section{B. Medical Treatment based on RFID}

In order to achieve the closed-loop system of the doctor's order, we advise the patient to wear RFID tag. We also stick RFID tag on medicine bottle and blood bag. In this way the individual medical staff can assure the accuracy and rapid identification of the patient, whether they have consciousness or not. With these tags, medical staff is able to collect and process the patient's information directly and correctly through the RFID reader and wireless network deployed [7]. The medical information includes all kinds of physiological indicators of the patient, such as medication, body temperature measurement times, nappy change times, baby sucking times and so on.

We propose an approach that provides a mechanism to prevent medical errors in the ward environment. The objective is to achieve an information system that supports the closed-loop medical identification and safety needs of the application.

\section{Architecture of RFID System}

RFID system consists of two levels of networks. The tag and reader construct the wireless communication network, staying in the front. The information network connects to the back-end application. The front-end deployment involves wireless network constructing and coordinating technology. The complex hardware architecture of RFID system and the mass feature of data put forward big challenge to the deployment of the system. On the other hand, when PDA moves in the wireless network environment, the coverage problem of wireless network may lead to instability of the connection, and influence the reliability and stability of the medical usage.

In order to solve these problems, we propose a RFID system according to the hospital environment. It will be more suitable for the hospital usage. The general structure is shown in Figure 2.

First, we need to determine the main performance index and constraints of RFID system architecture, such as RFID performance, signal interference and wireless coverage constraints.

Secondly, we analyze the RFID system architecture of service performance. The result is used in deploying RFID network system architecture.

Steps include

- Analyze the various properties of RFID tags (return loss, directivity, etc.). We choose the most suitable design according to the characteristics of ward.

- Analyze the various properties of RFID readers (read range, the fastest response time, etc.). Also choose the most suitable devices according to characteristics of ward.

- Design the layout of the system structure according to the situation of electromagnetic radiation and absorption in the ward environment of variety materials.

- Test the RFID tags, antennas and readers selected for this environment. Make sure to consider the moving direction, speed, number and other parameters.

- Select the network communication protocol. Communication is established between equipments and devices, between equipments and business logic modules, between the business logic modules and the upper layer applications of HIS.

- Evaluate the performance and effectiveness of the system architecture. Identify the possible bottlenecks and improve the usability of the system. It is the last but very important step.

\section{REAlization OF WiRELESS System BAsed ON RFID}

\section{A. Workflow}

Based on RFID technology, the real-time checking and identification of every step of the process is achieved [8]. With the unique identification of the patient, on the medicine bottle or the blood bag, double check on every step of the medical treatment is realized. The initial information gathering from the patient is obtained. And all the final data 
after process of doctor's order is written to HIS. Thus the entire closed loop of the process is built. With the data obtained, we can easily know who did what, and which step of the loop was completed and when it was done.

The closed-loop executive system of doctor's advice based on RFID is shown in Figure 3. In the ward environment, WLAN is deployed, and wireless AP is layout according to the PDA operating region demand. With the patient wearing RFID tag, the nurse carrying PDA with RFID reading function, they exchange information through the wireless network and HIS. The nurse identifies the patient according to his (her) RFID tag. At the same time, the information of treatment is double confirmed when doctor's advice is executed, and the double check steps are recorded through RFID system in real time. The information of when the doctor's advice was carried out, who carried it out, and the patient symptom data etc. are saved into HIS through PDA automatically.

\section{B. System Function}

Wireless System based on RFID extends the information system to the ill in bed. It is used by the nurse, supporting process of doctor's order [9]. We realize the whole lifecycle information management of doctor's order, and complete the medical closed-loop control. System functions include

- Scan the unique RFID tag to assure the patient's identification and medical data.

- Input and query laboratory sheet. Input physical information of the patient (such as blood pressure data, body temperature and pulse data, etc.). Edit the nursing information and the diagnostic message in the first page of the patient's record.

- Complete the operation of patient's in-department, changing-department, exchanging-bed and corresponding cancel handling.

- Finish checking and disannulling operation of the doctor's order. Realize the query and dispose of the doctor's order. The information of the medicine and the valuation item of the doctor's order are input into HIS.

- $\quad$ Use the calling function with IP-PHONE of Wireless Fidelity (Wi-Fi), which is convenient to the patient and medical staff.

- Accomplish the query of knowledge base, thus providing the correct, quick and flexible tool for achieving knowledge and technology needed.

\section{Hardware and Software}

Hardware of the System include

- $\quad$ RFID reader, model Compact Flash Reader KD801, Supporting ISO14443A(B), ISO 15693 standard

- PDA, model HP iPAQ hx2490c, Windows Mobile 5.0 Runtime Environment

- AP, model Gigabyte GN-A11G, IEEE 802.11g/802.11b protocol

- $\quad$ RFID tag writer, model Intellitag PM4i, $13.56 \mathrm{MHz}$ frequency, supporting ISO 15963 standard
Software is focused on the database and application development. Our database adopts the tables from HIS. Except for the main index of patient, the order form and price list, the comparison tables of patient identification, tables of the RFID tag and doctor's order are added.

Development Environment of Software is Microsoft Visual Studio, of which Smart Device Cab is adopted as the template. Microsoft .Net Framework 3.5 SDK (Software Development Kit) is used in development.

The login and function selection come first in the system. Then the user completes the closed-loop process of the doctor's order, or chooses the IP-PHONE communicating based on Wi-Fi. The knowledge base is used if query needed. After all operation finished user will logout from the system.

\section{CONCLUSION}

No matter how carefully designed and extensively tested, systems will always contain unanticipated vulnerabilities to meet the patients' needs. The more complex the system is, the more difficult to eradicate the vulnerabilities, and the system become increasingly complex. To address these problems, our system has correct identification of patients and support closed-loop process of medical treatment. Increasing medical security is an ambitious goal. We adopt usable technology to assure the accuracy and effectiveness of doctor's order process, to determine the responsibility of the nurse, to collect a lot of clinic medical data that is valuable to further experiment. Our closed-loop system based on RFID optimizes medical procedure and provides reliable data for hospital performance evaluation, thereby guarantees the quality of medical care and nursing. HIS based on RFID ensure us 3-rights: that is right person gets right treatment at the right time.

\section{REFERENCES}

[1] Courtney, K.L., Demiris, G., Alexander, G.L., "Information technology: changing nursing processes at the point-of-care”, Nursing Administration Quarterly, 2005,29(4): 315-322

[2] Kumiko Ohashi, "Safe Patient transfer system with mornitoring of location and vital signs”, Med Dent Sci , 2008,vol.55,pp.33-41.

[3] J.M.Bailey,W.M.Haddad,andT.Hayakawa, “Closed-loop control in clinical pharmacology: Paradigms,benefits and challenges”,in Proceedings of the 2004 American Control Conference, 2004,pp 2268-2277.

[4] A.M.Wicks,J.K.Visich,and S.Li, "Radio frequency identification Applications in hospital environments," Hospital Topics, vol.84, 2006,pp.3-8.

[5] H.A.NahasandJ.S.Deogun,"Radio frequency identification Applications in smart hospitals,"in Proceedings of the $20^{\text {th }}$ IEEE International Symposium on Computer-Based Medical Systems,2007,pp.337-342.

[6] A.Lahtela,"A short overview of the RFID technology in healthcare,"in $4^{\text {th }}$ International Conference on Systems and Networks Communications,2009,pp.165-169.

[7] Sangwan, R.S., R. G. Qiu, and D. Jessen,"Using RFID tags for tracking patients, charts and medical equipment within an integrated health delivery network," Proceedings of Networking, Sensing andControl, 2005 IEEE.pp. 1070- 1074

[8] European Committee for Standardization, “A Mobile E-health System based on Workflow Automation Tool”, Information Society RTD Standards Implementation Report, May, 2002. 
[9] Holzinger, A., K. Schwaberger, and M. Weitlaner, "Ubiquitous computing for hospital applications: RFID-applications to enable research in real-life environments," Proceedings of the 29th Annual
International Computer Software and Conference(COMPSAC'05), 2005,vol. 2, pp.19-20.
Applications

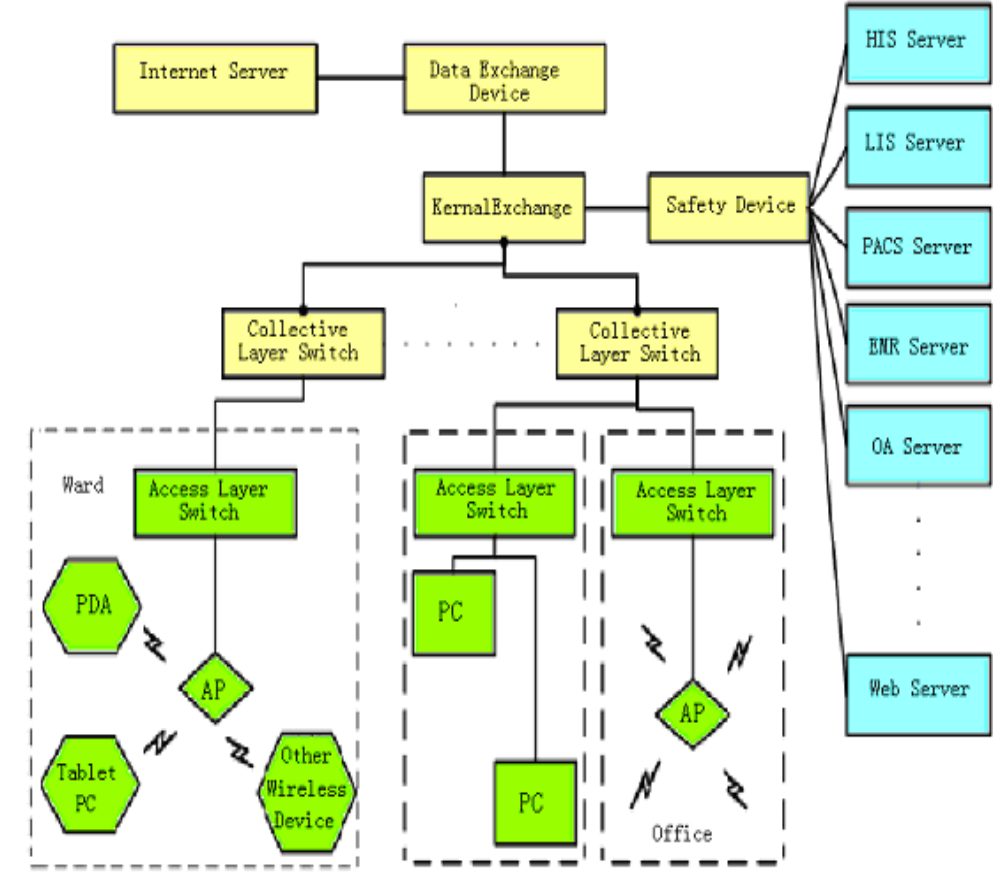

Figure 1 Wireless Network Structure

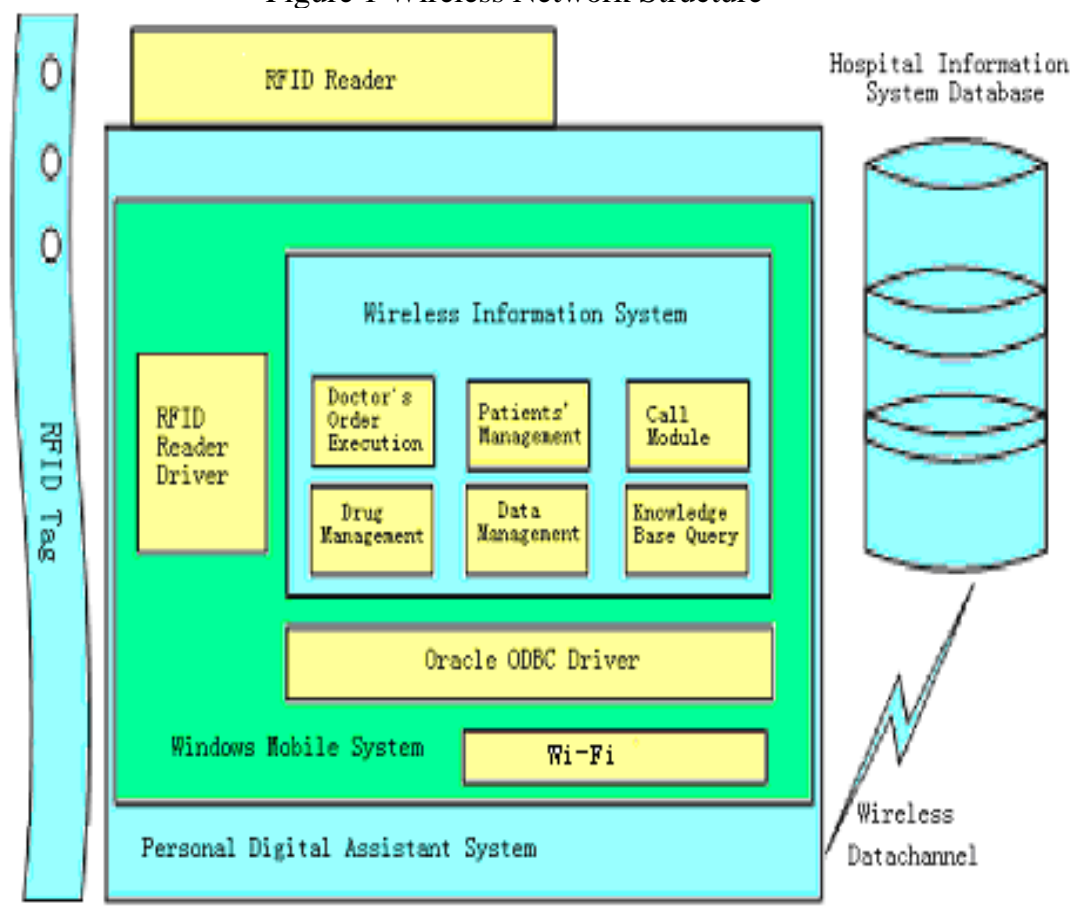

Figure 2 RFID System Architecture 


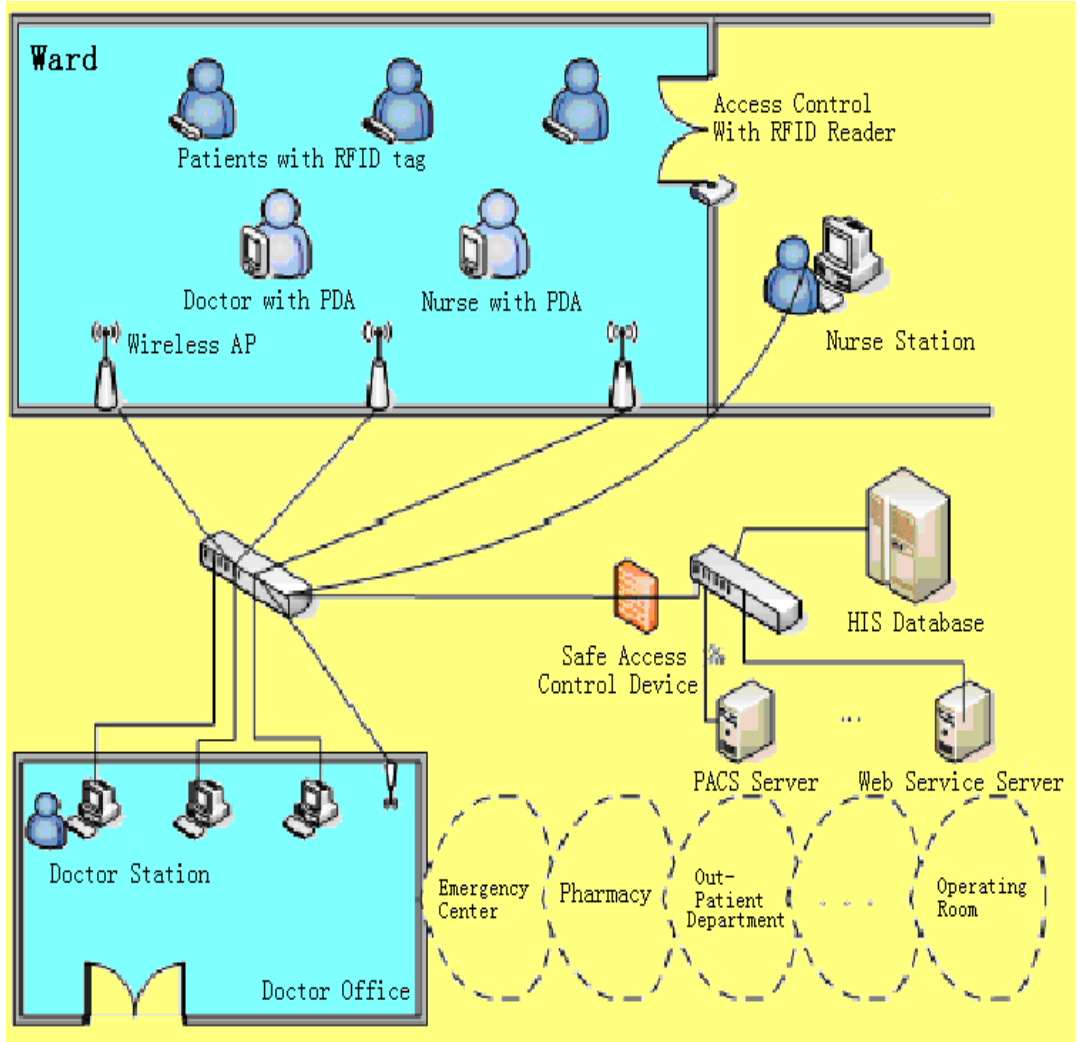

Figure 3 RFID Closed-loop System 